\title{
Backward Bifurcation in a Model for Vector Transmitted Disease
}

\author{
Hisashi Inaba*
}

\section{Introduction}

In mathematical models for the spread of infectious diseases, it is well known that there is a threshold phenomena: if the basic reproduction number $R_{0}$ is grater than one, the disease can invade into the susceptible host community, whereas it cannot if $R_{0}$ is less than one. The basic reproduction number is the average number of secondary cases produced by one infectious individual, during its total infective period, in a population that is in the disease-free steady state (see Anderson and May 1991, Diekmann and Heesterbeek 2000).

Mathematically speaking, the threshold phenomena implies that the diseasefree steady is locally asymptotically stable if $R_{0}<1$, it is unstable for $R_{0}>1$ and at $R_{0}=1$ an endemic steady state bifurcates from the disease-free steady state. In most epidemic models investigated so far, the bifurcation at $R_{0}=1$ is forward (supercritical), that is, for $R_{0}<1$ there is no endemic steady state and the disease-free steady state is globally asymptotically stable, and for $R_{0}>1$ the bifurcated endemic steady is unique and locally stable at least as long as it is small enough (near the bifurcation point), though we do not know what will happen for the endemic steady states away from the bifurcation point. It may lose its stability in various way (Cha, et al. 2000).

On the other hand, recently some authors have found epidemic models leading to backward bifurcations (subcritical bifurcation) at $R_{0}=1$ and stressed its important consequences for the control of infectious disease (see Hadeler and P. van den Driessche 1997, Kribs-Zaleta and J. X. Velasco-Hernández 2000, Kribs-Zaleta and M. Martcheva 2002).

In this note, we present a new epidemic mechanism leading to backward bifurcation of endemic steady states. We consider here a simple epidemic model for vector transmitted disease. The basic model can be seen as a simplified version of models for Chagas disease developed by Velasco-Hernández (1991, 1994) and Sekine (2002). Chagas disease or American trypanosomiasis caused

*Department of Mathematical Sciences, University of Tokyo, 3-8-1 Komaba Meguro-ku, Tokyo 153-8914 Japan; E-mail: inaba@ms.u-tokyo.ac.jp 
by the protozoan parasite Trypanosoma cruiz and transmitted by blood-feeding triatomine bugs, is a chronic, frequently fatal infection that is common in Latin America. There is so far no effective medical treatments and no vaccine. Though Chagas disease can be also transmitted by blood transfusion, organ transplants and the placenta (vertical transmission from mother to babies), here we only consider the vector transmission and neglect the variable infectivity since our purpose here is not to develop a realistic model for Chagas disease, but to show a possible mechanism leading backward bifurcation as simply as possible.

\section{The basic model}

Let us consider a fatal disease transmitted by a vector population. We divide the host population into two groups: $S(t)$ denotes the density of uninfected, but susceptible host population and $I(t)$ denotes the density of infected hosts where $t$ denotes time. The infected population is removed with the extra death rate due to disease $\gamma>0$. Then the total size of host population is given by $T(t):=$ $S(t)+I(t)$. Let $M(t)$ denote the density of susceptible vectors at time $t, V(t)$ the density of infected vectors at time $t$ and $U(t):=M(t)+V(t)$ the total density of vectors. Let $b_{1}\left(b_{2}\right)$ be the birth rate of host population (vectors), $\mu_{1}\left(\mu_{2}\right)$ the natural death rate of host population (vectors). For the vector population, we assume that there is no extra death rate due to infection.

For modeling vector transmitted disease, the most important ingredient is the formula of the force of transmission. Here we adopt a most simple assumption that is very common among traditional modeling of vector transmitted diseases like as malaria. The reader may refer to Esteva and Matias (2001) for another type of assumption of the force of infection.

Let $a$ be the number of bites per vector per unit time and $c_{1}$ be the proportion of infected bites that give rise to infection from infected vector to susceptible host. For simplicity, we assume that $a$ is a constant. Then the $V$ vector makes $a c_{1} V$ infectious bites of which a fraction $S / T$ are on susceptible hosts. That is, the number of new infection of hosts per unit time by vector transmission is given by $a c_{1} V(S / T)$.

Hence the force of infection (the probability per unit of time for a susceptible to become infected) for host population, denoted by $\lambda_{1}(t)$, is given by

$$
\lambda_{1}(t)=\alpha V(t) / T(t)
$$

where $\alpha:=a c_{1}$. By using similar argument, we know that the force of infection for vector population, denoted by $\lambda_{2}(t)$, is given by

$$
\lambda_{2}(t)=\beta I(t) / T(t),
$$

where $\beta:=a c_{2}$ and $c_{2}$ denotes the proportion of bites that give rise to infection from infected host to susceptible vector. 
Under the above assumption, our basic model for a vector transmitted disease can be formulated as follows:

$$
\begin{aligned}
& d S(t) / d t=b_{1}-\left(\mu_{1}+\lambda_{1}(t)\right) S(t), \\
& d I(t) / d t=\lambda_{1}(t) S(t)-\left(\mu_{1}+\gamma\right) I(t), \\
& d M(t) / d t=b_{2}-\left(\mu_{2}+\lambda_{2}(t)\right) M(t), \\
& d V(t) / d t=\lambda_{2}(t) M(t)-\mu_{2} V(t),
\end{aligned}
$$

If we add two equations (2.4c) and (2.4d) for vector population, we obtain a single equation for the total vector population $U=M+V$ as

$$
d U(t) / d t=b_{2}-\mu_{2} U(t) .
$$

It is easily seen that $U^{*}:=b_{2} / \mu_{2}$ is a globally stable steady state. Then in the following, we assume in advance that $M(t)+V(t)=b_{2} / \mu_{2}$ for all $t \geq 0$. Then it is easy to see that the solution of $(2.3 \mathrm{a})-(2.3 \mathrm{~d})$ starting from the region

$$
\Omega:=\left\{(S, I, M, V) \in \mathbf{R}_{+}^{4}: 0 \leq S+I \leq b_{1} / \mu_{1}, M+V=b_{2} / \mu_{2}\right\}
$$

stays in $\Omega$ for all $t>0$, that is, $\Omega$ is positively invariant with respect to the flow generated by the basic system $(2.3)$.

The system (2.3a)-(2.3d) has a trivial steady state (the disease-free steady state) as

$$
\left(S^{*}, I^{*}, M^{*}, V^{*}\right)=\left(b_{1} / \mu_{1}, 0, b_{2} / \mu_{2}, 0\right) .
$$

The dynamics of the initial invasion phase is described by the linearized system at the disease-free steady state. At the disease-free steady state, we can formulate the linearized system as follows:

$$
\left(\begin{array}{c}
\dot{I} \\
\dot{V}
\end{array}\right)=\left(\begin{array}{cc}
-\left(\mu_{1}+\gamma\right) & \alpha \\
\beta b_{2} \mu_{1} /\left(b_{1} \mu_{2}\right) & -\mu_{2}
\end{array}\right)\left(\begin{array}{c}
I \\
V
\end{array}\right),
$$

By calculating the eigenvalue, we can conclude that the disease-free steady state is locally asymptotically stable if $R_{0}<1$, whereas it is unstable if $R_{0}>1$, where the basic reproduction number $R_{0}$ is calculated as

$$
R_{0}=\frac{\alpha \beta b_{2} \mu_{1}}{b_{1} \mu_{2}^{2}\left(\mu_{1}+\gamma\right)} .
$$

By using the basic reproduction number, we can formulate the threshold condition for this vector transmitted disease as follows:

Proposition 2. 1 If $R_{0}<1$, then the disease-free steady state is locally asymptotically stable, and if $R_{0}>1$, it is unstable. Moreover, if 


$$
R_{0}<\frac{\mu_{1}}{\mu_{1}+\gamma},
$$

then the disease-free steady state is globally asymptotically stable.

Proof. The eigenvalues of the coefficient matrix of (2.5) are give as the solution of quadratic equation as

$$
\lambda^{2}+\left(\mu_{1}+\mu_{2}+\gamma\right) \lambda+\left(\mu_{1}+\gamma\right) \mu_{2}\left(1-R_{0}\right)=0,
$$

where $R_{0}$ is given by (2.6). Then if $R_{0}<1$, all eigenvalues have negative real parts, and if $R_{0}>1$ one of eigenvalue is positive. Therefore the first half of this statement is obvious. Let us proof the latter half. It follows from (2.3a) and (2.3b) that

$$
d T(t) / d t=b_{1}-\mu_{1} T(t)-\gamma I(t) \geq b_{1}-\left(\mu_{1}+\gamma\right) T(t) .
$$

Then we obtain

$$
T(t) \geq \frac{b_{1}}{\mu_{1}+\gamma}+\left(T(0)-\frac{b_{1}}{\mu_{1}+\gamma}\right) e^{-\left(\mu_{1}+\gamma\right) t} .
$$

Hence if $T(0) \geq b_{1} /\left(\mu_{1}+\gamma\right)$, it follows that $T(t) \geq b_{1} /\left(\mu_{1}+\gamma\right)$ for all $t \geq 0$. Even if $T(0)<b_{1} /\left(\mu_{1}+\gamma\right)$, the condition $T(t) \geq b_{1} /\left(\mu_{1}+\gamma\right)$ will be satisfied as time goes to infinity, so without loss of generality, we can assume that $T(0) \geq$ $b_{1} /\left(\mu_{1}+\gamma\right)$ holds in advance. Hence we can assume that $1 / T(t) \leq\left(\mu_{1}+\gamma\right) / b_{1}$.

Next let us define functions $B(t)$ and $C(t)$ as follows:

$$
B(t):=\lambda_{1}(t) S(t), \quad C(t)=: \lambda_{2}(t) M(t),
$$

that is, $B(t)$ denotes the number of newly infected hosts at time $t$ and $C(t)$ is the number of newly infected vectors at time $t$. From (2.3), we have the following expressions:

$$
\begin{aligned}
& S(t)=\frac{b_{1}}{\mu_{1}}+\left(S(0)-\frac{b_{1}}{\mu_{1}}\right) e^{-\mu_{1} t}-\int_{0}^{t} e^{-\mu_{1}(t-s)} B(s) d s, \\
& I(t)=I(0) e^{-\left(\mu_{1}+\gamma\right) t}+\int_{0}^{t} e^{-\left(\mu_{1}+\gamma\right)(t-s)} B(s) d s \\
& V(t)=V(0) e^{-\mu_{2} t}+\int_{0}^{t} e^{-\mu_{2}(t-s)} C(s) d s .
\end{aligned}
$$

Then we obtain a system of Volterra integral equations:

$$
\begin{aligned}
& B(t)=\alpha \frac{S(t)}{T(t)}\left(V(0) e^{-\mu_{2} t}+\int_{0}^{t} e^{-\mu_{2}(t-s)} C(s) d s\right), \\
& C(t)=\beta \frac{M(t)}{T(t)}\left(I(0) e^{-\left(\mu_{1}+\gamma\right) t}+\int_{0}^{t} e^{-\left(\mu_{1}+\gamma\right)(t-s)} B(s) d s\right) .
\end{aligned}
$$


By using inequalities $S / T \leq 1$ and $M / T \leq\left(\mu_{1}+\gamma\right) b_{2} /\left(b_{1} \mu_{2}\right)$ and if we insert (2.12) into (2.11), it follows that

$$
B(t) \leq G(t)+\int_{0}^{t} K(s) B(t-s) d s,
$$

where

$$
\begin{aligned}
K(s) & :=\frac{\alpha \beta\left(\mu_{1}+\gamma\right) b_{2}}{b_{1} \mu_{2}} \int_{0}^{s} e^{-\mu_{2}(s-\sigma)} e^{-\left(\mu_{1}+\gamma\right) \sigma} d \sigma \\
G(t) & :=\alpha V(0) e^{-\mu_{2} t}+\frac{\alpha \beta\left(\mu_{1}+\gamma\right) b_{2}}{b_{1} \mu_{2}} I(0) \int_{0}^{t} e^{-\mu_{2}(t-s)} e^{-\left(\mu_{1}+\gamma\right) s} d s .
\end{aligned}
$$

Therefore we conclude that $\lim _{t \rightarrow \infty} B(t)=0$ if

$$
\int_{0}^{\infty} K(s) d s=\frac{\alpha \beta b_{2}}{b_{1} \mu_{2}^{2}}=R_{0} \frac{\mu_{1}+\gamma}{\mu_{1}}<1 .
$$

Then (2.7) is a sufficient condition for global stability of the disease-free steady state.

\section{$3 \quad$ Backward bifurcation of endemic steady states}

Let us denote $S^{*}, I^{*}, M^{*}$ and $V^{*}$ as the stationary states of $S(t), I(t), M(t)$ and $V(t)$ respectively, and let $\lambda_{j}^{*},(j=1,2)$ be the force of infection corresponding to the stationary state. Then we have

$$
\left\{\begin{array}{l}
0=b_{1}-\lambda_{1}^{*} S^{*}-\mu_{1} S^{*} \\
0=\lambda_{1}^{*} S^{*}-\left(\mu_{1}+\gamma\right) I^{*} \\
0=b_{2}-\lambda_{2}^{*} M^{*}-\mu_{2} M^{*} \\
0=\lambda_{2}^{*} M^{*}-\mu_{2} V^{*}
\end{array}\right.
$$

Then it is easy to obtain the expression for steady states as follows:

$$
\left(S^{*}, I^{*}, M^{*}, V^{*}\right)=\left(\frac{b_{1}}{\mu_{1}+\lambda_{1}^{*}}, \frac{b_{1} \lambda_{1}^{*}}{\left(\mu_{1}+\lambda_{1}^{*}\right)\left(\mu_{1}+\gamma\right)}, \frac{b_{2}}{\mu_{2}+\lambda_{2}^{*}}, \frac{\lambda_{2}^{*} b_{2}}{\mu_{2}\left(\mu_{2}+\lambda_{2}^{*}\right)}\right),
$$

From relations $\lambda_{1}^{*}=\alpha V^{*} /\left(S^{*}+I^{*}\right)$ and $\lambda_{2}^{*}=\beta I^{*} /\left(S^{*}+I^{*}\right)$, we obtain equations as

$$
\begin{aligned}
\lambda_{1}^{*} & =\frac{\alpha b_{2}}{\mu_{2} b_{1}} \frac{\left(\mu_{1}+\lambda_{1}^{*}\right)\left(\mu_{1}+\gamma\right)}{\lambda_{1}^{*}+\mu_{1}+\gamma} \frac{\lambda_{2}^{*}}{\mu_{2}+\lambda_{2}^{*}}, \\
\lambda_{2}^{*} & =\frac{\lambda_{1}^{*} \beta}{\mu_{1}+\gamma+\lambda_{1}^{*}} .
\end{aligned}
$$

From (3.3) and (3.4), we can derive an equation satisfied by $\lambda_{1}^{*}$ as 


$$
\begin{aligned}
& \lambda_{1}^{*}=\frac{\alpha \beta b_{2}}{\mu_{2} b_{1}} \frac{\left(\mu_{1}+\lambda_{1}^{*}\right)\left(\mu_{1}+\gamma\right)}{\lambda_{1}^{*}+\mu_{1}+\gamma} \frac{\lambda_{1}^{*}}{\mu_{2}\left(\mu_{1}+\gamma\right)+\lambda_{1}^{*}\left(\mu_{2}+\beta\right)} \\
& =\lambda_{1}^{*} R\left(\lambda_{1}^{*}\right),
\end{aligned}
$$

where $R_{1}(\lambda)$ is a function defined by

$$
R(\lambda):=\frac{\alpha \beta b_{2}}{\mu_{2} b_{1}} \frac{\left(\mu_{1}+\lambda\right)\left(\mu_{1}+\gamma\right)}{\lambda+\mu_{1}+\gamma} \frac{1}{\mu_{2}\left(\mu_{1}+\gamma\right)+\lambda\left(\mu_{2}+\beta\right)} .
$$

By using the expression for steady states, we can decompose $R\left(\lambda_{1}^{*}\right)$ as

$$
R\left(\lambda_{1}^{*}\right)=\left[\alpha \cdot \frac{1}{\mu_{2}} \cdot \frac{S^{*}}{T^{*}}\right] \cdot\left[\frac{1}{\mu_{1}+\gamma} \cdot \beta \cdot \frac{U^{*}}{T^{*}} \cdot \frac{M^{*}}{U^{*}}\right],
$$

which shows that $R(\lambda)$ is the product of the average number of infected hosts produced by an infected vector during its infective period and the average number of infected vectors produced by an infected host during its infective period at the endemic steady state. That is, $R(\lambda)$ is no other than the reproduction number at the endemic steady state with the force of infection $\lambda$. Then we can prove the following bifurcation result for the endemic steady states:

Proposition 3.1 Let us define a number $D$ and $G$ as

$$
D:=\mu_{1}^{2}-\left(\mu_{1}+\gamma\right) G, \quad G:=\mu_{1}-\frac{\gamma \mu_{2}}{\mu_{2}+\beta} .
$$

If $G \geq 0$, then there exists a unique endemic steady state if and only if $R_{0}>1$. If $G<0$, then the following holds:

(1) If $R_{0} \geq 1$, there exists only one endemic steady state.

(2) If $R_{0}<1$ and $R\left(-\mu_{1}+\sqrt{D}\right)>1$, then there exists two endemic steady state.

(3) If $R_{0}<1$ and $R\left(-\mu_{1}+\sqrt{D}\right)=1$, then there exists only one endemic steady state.

(4) If $R_{0}<1$ and $R\left(-\mu_{1}+\sqrt{D}\right)<1$, then there is no endemic steady state.

Proof. Observe that

$$
R(\lambda)=\frac{\alpha \beta b_{2}\left(\mu_{1}+\gamma\right)}{\mu_{2} b_{1}}\left(1-\frac{\gamma}{\lambda+\mu_{1}+\gamma}\right) \frac{1}{\mu_{2}\left(\mu_{1}+\gamma\right)+\lambda\left(\mu_{2}+\beta\right)} .
$$

Then we obtain

$$
R^{\prime}(\lambda)=\frac{\alpha \beta b_{2}\left(\mu_{1}+\gamma\right)}{\mu_{2} b_{1}} \frac{-f(\lambda)}{\left(\mu_{1}+\gamma+\lambda\right)^{2}\left(\mu_{2}\left(\mu_{1}+\gamma\right)+\lambda\left(\mu_{2}+\beta\right)\right)^{2}},
$$

where 


$$
f(\lambda)=\left(\mu_{2}+\beta\right)\left(\lambda^{2}+2 \mu_{1} \lambda+\left(\mu_{1}+\gamma\right) G\right) .
$$

If $G \geq 0$, then $R^{\prime}(\lambda) \leq 0$ for all $\lambda>0$, so $R(\lambda)$ is monotone decreasing for $\lambda>$ 0 . Since

$$
R(0)=R_{0}=\frac{\alpha \beta b_{2} \mu_{1}}{b_{1} \mu_{2}^{2}\left(\mu_{1}+\gamma\right)}, \quad \lim _{\lambda \rightarrow \infty} R(\lambda)=0,
$$

then we know that the characteristic equation $R(\lambda)=1$ has only one positive root if and only if $R_{0}>1$. On the other hand, if $G<0$, then we have $D>0$ and

$$
R^{\prime}(\lambda)=-\frac{\alpha \beta b_{2}\left(\mu_{1}+\gamma\right)}{\mu_{2} b_{1}} \frac{\left(\lambda+\mu_{1}+\sqrt{D}\right)\left(\lambda+\mu_{1}-\sqrt{D}\right)}{(\mu+\gamma+\lambda)^{2}\left(\left(\mu_{2}\left(\mu_{1}+\gamma\right)+\lambda\left(\mu_{2}+\beta\right)\right)^{2}\right.} .
$$

Hence we know that $R(\lambda)$ has a unimodal pattern and it attains the maximum value at $\lambda=-\mu_{1}+\sqrt{D}>0$. Then the above statement follows.

Corollary 3.2 The backward bifurcation does not occur for our model if the extra death rate $\gamma$ due to the disease is zero.

From the above propositions, we know that a backward bifurcation of endemic steady states could occur at $R_{0}=1$ under the condition $G<0$, and we do not yet know what kind of parameter set satisfies the condition $R\left(-\mu_{1}+\right.$ $\sqrt{D}) \geq 1$. However, observe that

$$
R^{\prime}(0)=-\frac{\alpha \beta b_{2}\left(\mu_{2}+\beta\right) G}{\mu_{2}^{3} b_{1}\left(\mu_{1}+\gamma\right)^{2}} .
$$

Hence if $G<0$, then $R^{\prime}(0)>0$, and it is easy to see that if $R_{0}<1$ and $\mid R_{0}-$ $1 \mid$ is small enough, there exists two endemic steady state.

On the other hand, if we note that the equation $R(\lambda)=1$ can be reduced to a quadratic equation

$$
g(\lambda):=\left(\mu_{2}+\beta\right) \lambda^{2}+H \lambda+\left(\mu_{1}+\gamma\right)^{2} \mu_{2}\left(1-R_{0}\right)=0,
$$

where $H$ is given by

$$
H:=\left(\mu_{1}+\gamma\right)\left(2 \mu_{2}+\beta-\frac{\alpha \beta b_{2}}{\mu_{2} b_{1}}\right) .
$$

Since $g^{\prime}(0)=H$ and $g(0)=\left(\mu_{1}+\gamma\right)^{2} \mu_{2}\left(1-R_{0}\right)$, by graphical consideration we can easily obtain the following another formulation:

Proposition 3.3 Let us define the discriminant $E$ of (3.6) as

$$
E:=H^{2}-4\left(\mu_{1}+\gamma\right)^{2}\left(\mu_{2}+\beta\right) \mu_{2}\left(1-R_{0}\right) .
$$

Then the following holds: 
(1) If $R_{0}>1$, there is only one endemic steady state,

(2) If $R_{0}=1$, there is only one endemic steady state when $H<0$, and there is no endemic steady state when $H \geq 0$,

(3) If $R_{0}<1$, there is no endemic steady state when $H \geq 0$. If $H<0$, there are two endemic steady states when $E>0$, there is only one endemic steady state when $E=0$ and there is no endemic steady state when $E<$ 0 .

Corollary 3. 4 Suppose that

$$
2+\frac{\beta}{\mu_{2}}<\frac{\alpha \beta b_{2}}{\mu_{2}^{2} b_{1}}<2+\frac{\beta}{\mu_{2}}+2 \sqrt{1+\frac{\beta}{\mu_{2}}} .
$$

Then the backward bifurcation occurs at $R_{0}=1$.

Proof. First observe that under the condition (3.7), we have

$$
H=\mu_{2}\left(\mu_{1}+\gamma\right)\left(2+\frac{\beta}{\mu_{2}}-\frac{\alpha \beta b_{2}}{\mu_{2}^{2} b_{1}}\right)<0 .
$$

Let us choose $R_{0}$ as a bifurcation parameter. Consider $\gamma$ as a function of $R_{0}$ and let us fix other parameters. Observe that

$$
\gamma=\frac{\alpha \beta b_{2} \mu_{1}}{b_{1} \mu_{2}^{2}} \frac{1}{R_{0}}-\mu_{1} .
$$

Then $R_{0}$ moves from zero to $\alpha \beta b_{2} /\left(b_{1} \mu_{2}^{2}\right), \gamma$ decreases monotonically from $\infty$ to zero. Moreover note that $E=0$ when $R_{0}=R_{0}^{*}$, where

$$
R_{0}^{*}:=1-\frac{\mu_{2}}{4\left(\mu_{2}+\beta\right)}\left(2+\frac{\beta}{\mu_{2}}-\frac{\alpha \beta b_{2}}{\mu_{2}^{2} b_{1}}\right)^{2},
$$

which is positive under the condition (3.7). Then we obtain the following table:

\begin{tabular}{|c|cccc|}
\hline$R_{0}$ & 0 & $R_{0}^{*}$ & 1 & $\alpha \beta b_{2} /\left(\mu_{2}^{2} b_{1}\right)$ \\
\hline$\gamma$ & $\infty$ & & $\mu_{1}\left(\alpha \beta b_{2} /\left(\mu_{2}^{2} b_{1}\right)-1\right)$ & 0 \\
\hline$E$ & $E<0$ & 0 & $E>0$ & \\
\hline
\end{tabular}

Therefore we know that for $R_{0}^{*}<R_{0}<1$, there exist two endemic steady states, and the backward bifurcation occurs at $R_{0}=1$. 


\section{Discussion}

We have developed a mathematical model for vector transmitted disease with fatality, and calculated the basic reproduction number $R_{0}$ to show that the disease can invade into the susceptible population and unique endemic steady state exists if $R_{0}>1$, whereas the disease dies out if $R_{0}$ is small enough. We also proved that depending on parameters, the backward bifurcation of endemic steady state can occur, so even if $R_{0}<1$, there could exist endemic steady states. In our modeling, existence of disease-induced death rate plays a crucial role for the existence of a backward bifurcation.

The presence of a backward bifurcation has practically important consequences for the control of infectious diseases. If the bifurcation of endemic state at $R_{0}=1$ is forward one, the size of infected population will be approximately proportional to the difference $\left|R_{0}-1\right|$. On the other hand, in a system with a backward bifurcation, the endemic steady state that exists for $R_{0}$ just above one could have a large infectious population, so the result of $R_{0}$ rising above one would be a drastic change in the number of infecteds. Conversely, reducing $R_{0}$ back below one would not eradicate the disease, as long as its reduction is not sufficient. That is, if the disease is already endemic, in order to eradicate the disease, we have to reduce the basic reproduction number so far that it enters the region where the disease-free steady state is globally asymptotically stable and there is no endemic steady state.

In this note, we have only discussed existence of backward bifurcation of endemic steady states, to consider their stability is a future problem. From general principle of subcritically bifurcated steady state solutions, we could expect that if $R_{0}<1$ and there are two endemic steady states, the steady state corresponding to the smaller force of infection is unstable, and the other steady state is locally stable. This kind of stability arguments will be shown in a separate paper.

\section{References}

[1] R. M. Anderson and R. M. May (1991), Infectious Diseases of Humans: Dynamics and Control, Oxford UP, Oxford.

[2] Y. Cha, M. Iannelli and F. A. Milner (2000), Stability change of an epidemic model, Dyn. Sys. Appl. 9: 361-376.

[3] O. Diekmann and J.A.P. Heesterbeek (2000), Mathematical Epidemiology of Infectious Diseases: Model Building, Analysis and Interpretation, John Wiley and Sons, Chichester.

[4] L. Esteva and M. Matias (2001), A model for vector transmitted diseases with saturation incidence, J. Biol. Sys. 9(4): 235-245. 
[5] K. P. Hadeler and P. van den Driessche (1997), Backward bifurcation in epidemic control, Math. Biosci. 146: 15-35.

[6] C. M. Kribs-Zaleta and J. X. Velasco-Hernández (2000), A simple vaccination model with multiple endemic states, Math. Biosci. 164: 183-201.

[7] C. M. Kribs-Zaleta and M. Martcheva (2002), Vaccination strategies and backward bifurcation in an age-since-infection structured model, Math. Biosci. 177/178: 317-332.

[8] H. Sekine (2002), A Model for Chagas Disease Continuously Depending on the Duration of Infection, M.A.Thesis, University of Tokyo. [in Japanese]

[9] J. X. Velasco-Hernández (1991), An epidemiological model for the dynamics of Chagas' disease, Biosystem 26: 127-134.

[10] J. X. Velasco-Hernández (1994), A model for Chagas disease involving transmission by vectors and blood transfusion, Theor. Popul. Biol. 46: 1-31. 